\title{
Generalized effective mode volume for leaky optical cavities
}

\author{
Kristensen, Philip Trøst; Van Vlack, C.; Hughes, S.
}

Published in:

Optics Letters

Link to article, DOI:

10.1364/OL.37.001649

Publication date:

2012

Document Version

Publisher's PDF, also known as Version of record

Link back to DTU Orbit

Citation (APA):

Kristensen, P. T., Van Vlack, C., \& Hughes, S. (2012). Generalized effective mode volume for leaky optical cavities. Optics Letters, 37(10), 1649-1651. https://doi.org/10.1364/OL.37.001649

\section{General rights}

Copyright and moral rights for the publications made accessible in the public portal are retained by the authors and/or other copyright owners and it is a condition of accessing publications that users recognise and abide by the legal requirements associated with these rights.

- Users may download and print one copy of any publication from the public portal for the purpose of private study or research.

- You may not further distribute the material or use it for any profit-making activity or commercial gain

- You may freely distribute the URL identifying the publication in the public portal

If you believe that this document breaches copyright please contact us providing details, and we will remove access to the work immediately and investigate your claim. 


\title{
Generalized effective mode volume for leaky optical cavities
}

\author{
P. T. Kristensen, ${ }^{1,2, *}$ C. Van Vlack, ${ }^{2}$ and S. Hughes ${ }^{2}$ \\ ${ }^{1}$ DTU Fotonik, Technical University of Denmark, DK-2800 Kgs. Lyngby, Denmark \\ ${ }^{2}$ Department of Physics, Queen's University, Ontario, K7L 3N6, Canada \\ *Corresponding author: ptkr@fotonik.dtu.dk
}

Received February 21, 2012; revised March 6, 2012; accepted March 6, 2012; posted March 6, 2012 (Doc. ID 163382); published May 9, 2012

\begin{abstract}
We show explicitly how the commonly adopted prescription for calculating effective mode volumes is wrong and leads to uncontrolled errors. Instead, we introduce a generalized mode volume that can be easily evaluated based on the mode calculation methods typically applied in the literature, and which allows one to compute the Purcell effect and other interesting optical phenomena in a rigorous and unambiguous way. (c) 2012 Optical Society of America OCIS codes: $000.3860,230.5750$.
\end{abstract}

Optical microcavities are inherently dissipative and are typically characterized by a quality factor, or $Q$-value, describing the relative energy loss per cycle as well as an effective mode volume, $V_{\text {eff }}$, which measures the spatial confinement of light in the cavity [1]. Cavities with high $Q$-values and small mode volumes provide enhanced light-matter interaction and are of both fundamental and technological interest. Effective mode volumes are ubiquitous in physics and connect to a wide range of optical phenomena. As a striking example of the use of mode volumes, an emitter at the field antinode in an optical cavity will experience a medium-enhanced radiation rate relative to that in a homogeneous medium given by the so-called Purcell factor [2]

$$
F_{\mathrm{P}}=\frac{3}{4 \pi^{2}}\left(\frac{\lambda_{\mathrm{c}}}{n_{\mathrm{c}}}\right)^{3}\left(\frac{Q}{V_{\mathrm{eff}}}\right),
$$

where $\lambda_{\mathrm{c}}$ is the free space wavelength, and $n_{\mathrm{c}}$ is the material refractive index at the field antinode $\mathbf{r}_{c}$. Mode volumes are often attributed to the physically appealing idea of a single cavity mode. However, in spite of the fact that cavity modes are widely used in the literature, more often than not there is a disturbing lack of a precise definition, and their mathematical properties therefore remain unspecified. The name might suggest that cavity modes are localized and vanish at large distances, similar to the bound states of the hydrogen atom. In fact, the opposite is true-for any finite $Q$, the cavity modes necessarily diverge exponentially at sufficiently large distances. This effectively renders the calculation of an effective mode volume nontrivial. In particular, defining $\epsilon_{\mathrm{r}}(\mathbf{r})$ as the relative permittivity and $\tilde{\mathbf{f}}_{\mathrm{c}}(\mathbf{r})$ as the cavity mode, the common (normal mode) prescription

$$
V_{\mathrm{eff}}^{\mathrm{N}}=\int_{V} \frac{\epsilon_{\mathrm{r}}(\mathbf{r})\left|\tilde{\mathbf{f}}_{\mathrm{c}}(\mathbf{r})\right|^{2}}{\left.\epsilon_{\mathrm{r}}\left(\mathbf{r}_{\mathrm{c}}\right) \tilde{\mathbf{f}}_{\mathrm{c}}\left(\mathbf{r}_{\mathrm{c}}\right)\right|^{2}} \mathrm{~d} \mathbf{r}
$$

is inapplicable because the integral diverges. For high- $Q$ cavities, regularization of the integral in Eq. (2) by introducing a cut-off has provided good correspondence with experimental results [3,4], but the mathematical basis and the limits of such an approach remain unclear.
In this Letter, we introduce a generalized mode volume, which is defined in a precise and unambiguous way for cavities with arbitrary $Q$ and which recovers Eq. (2) in the limit of infinite $Q$. For general resonant structures, this provides the proper theoretical framework for the Purcell factor and other optical phenomena that may be interpreted in terms of mode volumes. In particular, the generalized mode volume applies also to structures with complex permittivity for which the limited validity of Eq. (2) has recently been pointed out [5]. We first argue that the term "cavity mode" can only be meaningfully defined as a solution to the wave equation with outgoing wave boundary conditions. This definition renders the cavity modes identical to the so-called quasinormal modes [] ], and we show how this definition complies with two different numerical calculation methods including finite-difference time-domain (FDTD). In particular, we elucidate how the modes from both calculation methods show an exponential divergence. Figure 1 shows a sketch of an example cavity along with the mode profile. For this cavity mode, we show explicitly how the integral in Eq. (2) diverges as a function of the integration volume $V$, and we emphasize that this will be the case for all cavities with a finite $Q$. For high- $Q$ cavities, however, the divergence in the cavity modes is slow and may not be discernible in practical calculations due to limited numerical accuracy.

The electric field satisfies the wave equation with general solutions of the form $\mathbf{E}(\mathbf{r}, t)=\mathbf{E}(\mathbf{r}, \omega) \exp \{-i \omega t\}$. The position-dependent field $\mathbf{E}(\mathbf{r}, \omega)$ solves the equation

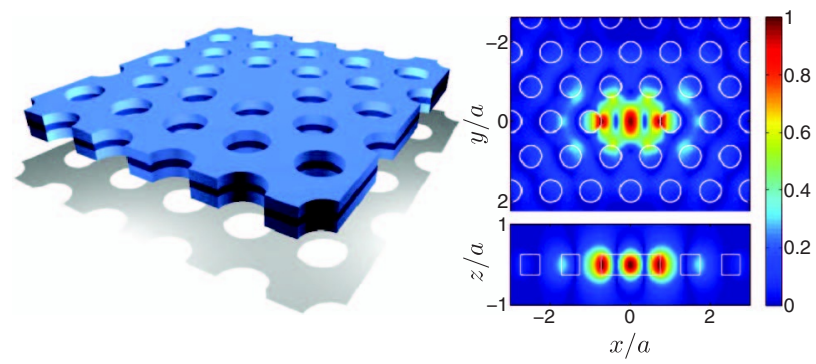

Fig. 1. (Color online) Sketch of a photonic crystal (lattice constant $a$ ) in a membrane of high refractive index. A defect cavity is formed by the omission of a single hole. Right: absolute value of the cavity mode in the planes $z=0$ (top) and $y=0$ (bottom). 


$$
\nabla \times \nabla \times \mathbf{E}(\mathbf{r}, \omega)-k_{0}^{2} \epsilon_{\mathrm{r}}(\mathbf{r}) \mathbf{E}(\mathbf{r}, \omega)=0,
$$

where $k_{0}=\omega / c$ is the ratio of the angular frequency to the speed of light. We define the cavity modes as the solutions to Eq. (3) with outgoing wave boundary conditions (the Sommerfeld radiation condition [7]). This choice of boundary conditions renders the problem non-Hermitian with a discrete spectrum. In order to distinguish from the normal modes of Hermitian eigenvalue problems, we denote the vector eigenfunctions with a tilde as $\tilde{\mathbf{f}}_{\mu}(\mathbf{r})$. The corresponding eigenfrequencies $\tilde{\omega}_{\mu}=\tilde{\omega}_{\mu}^{\mathrm{R}}+i \tilde{\omega}_{\mu}^{\mathrm{I}}$ are in general complex with $\tilde{\omega}_{\mu}^{\mathrm{I}}<0$, and the $Q$-value is obtained immediately as $Q=-\tilde{\omega}_{\mu}^{\mathrm{R}} / 2 \tilde{\omega}_{\mu}^{\mathrm{I}}$. This, together with the examples below, show that the above definition is completely consistent with the expected properties of cavity modes. At large distances, the boundary conditions force the modes to behave as outgoing waves of the form $\tilde{\mathbf{f}}(\mathbf{r}) \propto \exp \left(i k_{0} r\right) / \sqrt{r}$ [two-dimensional (2D)] and $\tilde{\mathbf{f}}(\mathbf{r}) \propto \exp \left(i k_{0} r\right) / r$ [threedimensional (3D)], where $r=|\mathbf{r}|$, and since $k_{0}=$ $k_{0}^{\mathrm{R}}+i k_{0}^{\mathrm{I}}$ with $k_{0}^{\mathrm{I}}<0$, they diverge exponentially as $r \rightarrow \infty$. Despite their divergence, the cavity modes may be normalized as []]

$$
\begin{aligned}
\left\langle\left\langle\tilde{\mathbf{f}}_{\mu} \mid \tilde{\mathbf{f}}_{\lambda}\right\rangle\right\rangle= & \lim _{V \rightarrow \infty} \int_{V} \epsilon_{\mathrm{r}}(\mathbf{r}) \tilde{\mathbf{f}}_{\mu}(\mathbf{r}) \cdot \tilde{\mathbf{f}}_{\lambda}(\mathbf{r}) \mathrm{d} \mathbf{r} \\
& +i \frac{\sqrt{\epsilon_{\mathrm{r}}} c}{\tilde{\omega}_{\mu}+\tilde{\omega}_{\lambda}} \int_{\partial V} \tilde{\mathbf{f}}_{\mu}(\mathbf{r}) \cdot \tilde{\mathbf{f}}_{\lambda}(\mathbf{r}) \mathrm{d} \mathbf{r}=\delta_{\mu, \lambda},
\end{aligned}
$$

where $\partial V$ denotes the border of the volume $V$. The limit $V \rightarrow \infty$ is calculated in practice by increasing the volume to obtain convergence. For the systems that we investigate below (and for all systems that we have investigated), the convergence is remarkably fast. For very low- $Q$ cavities, however, the convergence is nontrivial due to the exponential divergence of the modes that may cause the inner product to oscillate around the proper value as a function of calculation domain size. The implicit assumption behind the notion of a cavity mode is that one mode $\tilde{\mathbf{f}}_{\mu}=\tilde{\mathbf{f}}_{\mathrm{c}}$ dominates the expansion of the electromagnetic Green's tensor in the cavity []. One can use this assumption and the normalization in Eq. (4) to recover Eq. (1) with the effective mode volume

$$
\frac{1}{V_{\mathrm{eff}}^{\mathrm{Q}}}=\operatorname{Re}\left\{\frac{1}{v_{\mathrm{Q}}}\right\}, \quad v_{\mathrm{Q}}=\frac{\left\langle\left\langle\tilde{\mathbf{f}}_{\mathrm{c}} \mid \tilde{\mathbf{f}}_{\mathrm{c}}\right\rangle\right\rangle}{\epsilon_{\mathrm{r}}\left(\mathbf{r}_{\mathrm{c}}\right) \tilde{\mathbf{f}}_{\mathrm{c}}^{2}\left(\mathbf{r}_{\mathrm{c}}\right)},
$$

where the generalized mode volume $v_{\mathrm{Q}}=v_{\mathrm{Q}}^{\mathrm{R}}+i v_{\mathrm{Q}}^{\mathrm{I}}$ is complex in general. This prescription provides a direct and unambiguous way of calculating the effective mode volume for arbitrary cavities.

For calculations of cavity modes in general structures, the currently most popular option within the photonics community is arguably to apply FDTD with perfectly matched layers to calculate the modes as the resonant fields that are excited by an initial short input pulse [8]. Another option is to calculate the cavity modes from a Fredholm type equation of the form

$$
\mathbf{E}(\mathbf{r}, \omega)=\left(\frac{\omega}{c}\right)^{2} \int_{V} \mathbf{G}^{\mathrm{B}}\left(\mathbf{r}, \mathbf{r}^{\prime}, \omega\right) \Delta \epsilon\left(\mathbf{r}^{\prime}\right) \mathbf{E}\left(\mathbf{r}^{\prime}, \omega\right) \mathrm{d} \mathbf{r}^{\prime},
$$

where $\Delta \epsilon(\mathbf{r})=\epsilon_{\mathrm{r}}(\mathbf{r})-\epsilon_{\mathrm{B}}$ and $\mathbf{G}^{\mathrm{B}}\left(\mathbf{r}, \mathbf{r}^{\prime}, \omega\right)$ is the electromagnetic Green's tensor in the background medium of permittivity $\epsilon_{\mathbf{B}}[\underline{9}$ ]. For practical solutions of Eq. (6), we use the expansion technique of [10] with an additional iteration of $k_{0}$ to make the solution self-consistent.

We first consider a 2D finite-sized hexagonal crystallite of high-index rods in air with a single missing rod in the center. The rods have relative permittivity $\epsilon_{\mathrm{r}}=11.4$ and radius $R=0.15 a$, where $a$ is the lattice constant, and we focus on out of plane polarization. The $Q$-value of the cavity depends on the number of rod layers $N$, and for the case of $N=1$, the top panel in Fig. 2 shows the agreement between the two independent methods for calculating the cavity modes. In particular, both methods clearly pick up the divergence in the field at large distances. Figure 3 shows, as a function of the size of the calculation domain, the effective mode volume in Eq. (5) along with the common definition in Eq. (2). Whereas $V_{\text {eff }}^{\mathrm{Q}}$ converges quickly to the limiting values, $V_{\text {eff }}^{\mathrm{N}}$ clearly increases with the size of the calculation domain.

The initial linear divergence in $V_{\text {eff }}^{\mathrm{N}}$ with the size of the normalization domain derives from the small but nonzero field immediately outside the crystallite; cf. Fig. 2 . At larger $R$, the field, and hence $V_{\text {eff }}^{\mathrm{N}}$, diverges exponentially. For increasing $Q$, the linear divergence with domain size becomes less pronounced, suggesting how the two formalisms provide the same result for infinite $Q$.
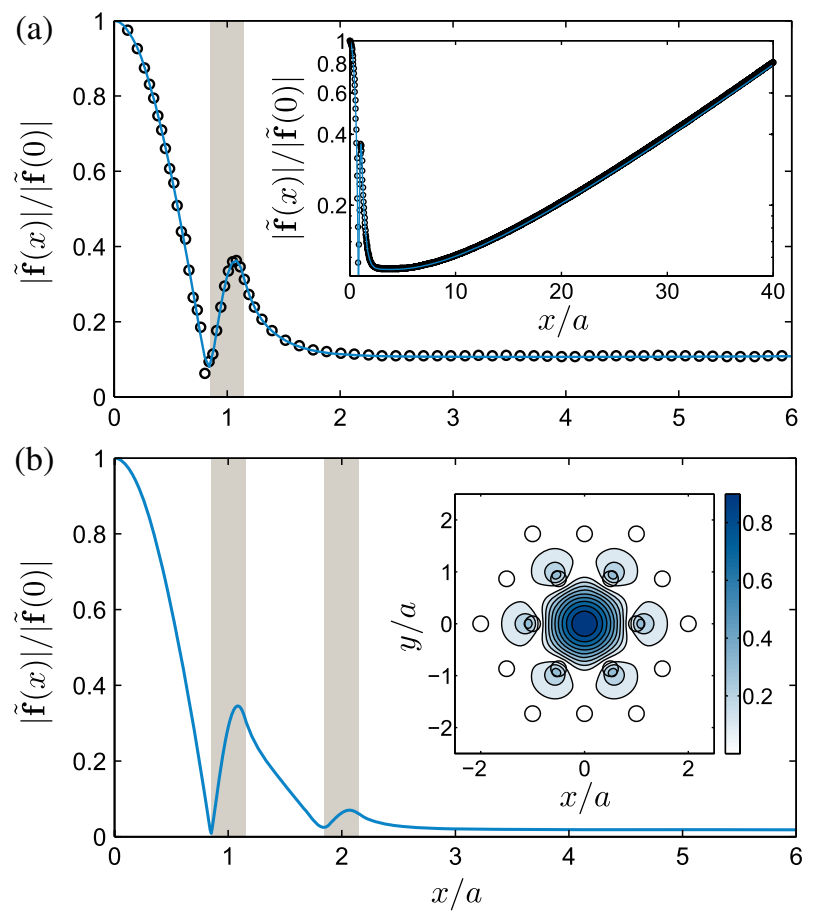

Fig. 2. (Color online) (a) Field along the $x$-axis of the cavity mode in the $2 \mathrm{D}$ crystallite for the case of $N=1$. Blue solid line shows the Fredholm type solution, and black circles show the calculation using FDTD. Inset shows long distance behavior on a logarithmic scale. (b) Field along the $x$-axis of the cavity mode for the case of $N=2$. Inset shows the field distribution in the $x y$-plane. 


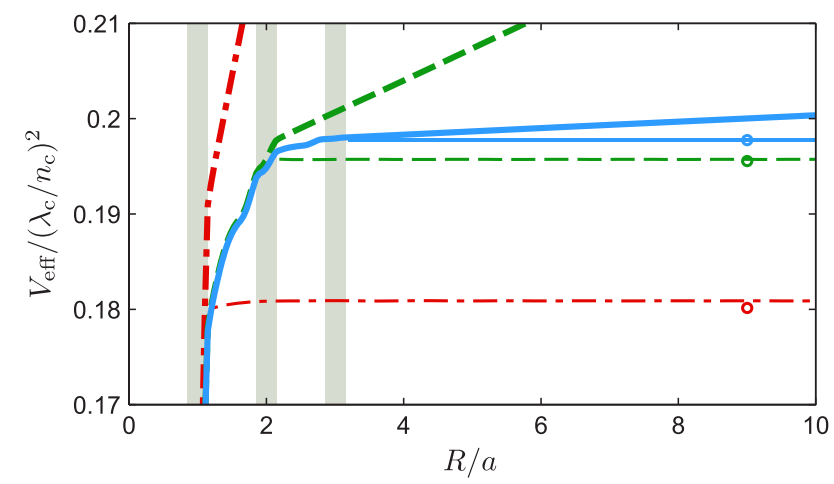

Fig. 3. (Color online) Effective mode volumes $V_{\text {eff }}^{\mathrm{N}}$ (thick lines) and $V_{\mathrm{eff}}^{\mathrm{Q}}$ (thin lines) for $N=1$ (red dash-dotted), $N=$ 2 (green dashed), and $N=3$ (blue solid) as a function of radius $R$ of the calculation domain. Circles indicate reference mode volumes $V_{\text {eff }}^{\text {tot }}$ from independent Green's tensor calculations [10].

Next, for a practical 3D example, we consider an infinite photonic crystal membrane $\left(\epsilon_{\mathrm{r}}=12\right)$ of thickness $h=0.5 a$ and hole radius $r=0.275 a$. A single air hole is omitted to create a cavity, and Fig. 1 shows the supported cavity mode calculated with FDTD [11]. For this cavity mode, Fig. $\underline{4}$ shows both $V_{\mathrm{eff}}^{\mathrm{Q}}$ and $V_{\mathrm{eff}}^{\mathrm{N}}$ as a function of calculation domain size. At the cavity mode frequency, which is within the in-plane photonic bandgap, the photonic crystal prevents in-plane propagation, and therefore the only way for the field to leak out of the cavity is in the $z$-direction. This means that both $V_{\text {eff }}^{\mathrm{Q}}$ and $V_{\text {eff }}^{\mathrm{N}}$ converge quickly as a function of width and depth of the calculation domain, and we therefore focus on the variation in the effective mode volumes with the height of the calculation domain. As in $2 \mathrm{D}$, the data shows a fast convergence of $V_{\mathrm{eff}}^{\mathrm{Q}}$, while $V_{\mathrm{eff}}^{\mathrm{N}}$ clearly diverges, illustrating why the use of Eq. (2) is wrong.

Finally, we compare the calculated mode volumes to independent calculations of the medium-enhanced radiation rate based on the electromagnetic Green's tensor $[8,10]$. In this case, Eq. (1) and the corresponding $2 \mathrm{D}$ ana$\log$ each defines a total effective mode volume, $V_{\text {eff }}^{\text {tot }}$, which is not limited by the single mode approximation. For each of the cavities, $V_{\text {eff }}^{\text {tot }}$ is indicated with a circle in Fig. 3. The estimated maximum absolute error in these calculations is less than $0.0003\left(\lambda_{\mathrm{c}} / n_{\mathrm{c}}\right)^{2}$. The observable discrepancies for $N=1$ and $N=2$ stem from the single mode approximation and indicate the limited validity of the Purcell formula for very low- $Q$ cavities. In Fig. $\underline{4}$, the Green's tensor was calculated with FDTD as the response to an input dipole source at three different domain sizes and with estimated error bars as indicated. These independent calculations confirm that Eq. (5) not only is unambiguous, but also leads to the correct value.

In conclusion, we have shown that for leaky optical cavities, the common prescription for the effective mode volume in Eq. (2) is wrong. The source of confusion can be traced to a lack of distinction between different types of modes. We argue that the term "cavity mode" should

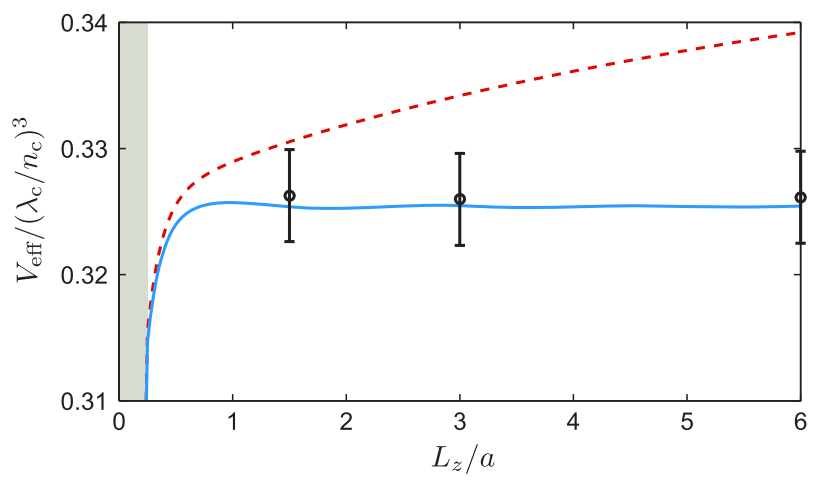

Fig. 4. (Color online) Effective mode volume $V_{\text {eff }}^{\mathrm{N}}$ (red dashed) and $V_{\text {eff }}^{\mathrm{Q}}$ (blue solid) for the cavity in Fig. 1 as a function of height of the calculation domain $L_{z}$. Circles indicate reference mode volumes $V_{\text {eff }}^{\text {tot }}$ derived from independent Green's tensor calculations [].

be properly defined as a solution to Eq. (3) with outgoing wave boundary conditions. This changes the properties of the eigenvalue problem so that common results from Hermitian eigenvalue analysis do not apply. In particular, the cavity modes have complex frequencies and exhibit an inherent divergence at long distances, which makes the calculation of an effective mode volume nontrivial. Using a normalization that carefully accounts for the long distance behavior, it is possible to define a generalized mode volume in a direct and unambiguous way. In practical calculations, this in turn provides the correct effective mode volume for use in Eq. (1) in a straightforward way using exactly the same cavity modes that are typically computed for mode volume calculations.

This work was supported by the National Sciences and Engineering Research Council of Canada, the Villum Kann Rasmussen Center of Excellence on Nanophototonics for Terabit Communications (NATEC), and The Danish Council for Independent Research (FTP 10-093651).

\section{References}

1. K. J. Vahala, Nature 424, 839 (2003).

2. E. M. Purcell, Phys. Rev. 69, 674 (1946).

3. T. J. A. Kippenberg, "Nonlinear optics in ulta-high-Q whispering-gallery optical microcavities," Ph.D. thesis, (Caltech, 2004).

4. K. Srinivasan, P. E. Barclay, M. Borselli, and O. Painter, Phys. Rev. B 70, 081306R (2004).

5. A. F. Koenderink, Opt. Lett. 35, 4208 (2010).

6. K. M. Lee, P. T. Leung, and K. M. Pang, J. Opt. Soc. Am. B 16, 1409 (1999).

7. P. Martin, Multiple Scattering (Cambridge, 2006).

8. P. Yao, V. S. C. Manga Rao, and S. Hughes, Laser Photon. Rev. 4, 499 (2010).

9. O. J. F. Martin and N. B. Piller, Phys. Rev. E 58, 3909 (1998).

10. P. T. Kristensen, P. Lodahl, and J. Mørk, J. Opt. Soc. Am. B 27, 228 (2010).

11. Lumerical FDTD Solutions: www.lumerical.com. 